\title{
PRODUÇĀO DE FRUTOS DE ESPÉCIES DA FLORESTA DE VÁRZEA DA AMAZÔNIA CENTRAL IMPORTANTES NA ALIMENTAÇÃO DE PEIXES
}

\author{
Lúcia Alencar MAIA ${ }^{1}$; Fiorella Perotti $\mathrm{CHALCO}^{2}$
}

RESUMO - Com o objetivo de quantificar a contribuição que quatro espécies, Cassia leiandra Benth., Crescentia amazonica Ducke, Macrolobium acacifolium (Benth.) Benth. e Vitex cymosa Bert. ex Spreng., oferecem através de seus frutos à alimentação dos peixes, foram marcados, aleatoriamente, dez indjvíduos adultos destas quatro espécies da floresta de várzea na ilha da Marchantaria - Amazônia Central. O período de maior produção de frutos dá-se no período do alto nível das águas, entre janeiro e maio. A espécie Cassia leiandra apresentou a maior produção com $1.836 \mathrm{~kg} / \mathrm{ha}$ e amplo consumo pela população local. Os principais peixes consumidores de frutos são tambaqui (Colossoma macropomum), matrinxã (Brycon cephalus), pirapitinga (Piaractes brachyponus), pirarara, (Phractocephalus hemivioptenus), bacu (Lithodosas torsalis, Megalodoras sp.), pacu (Mylossoma sp., Myleus sp., Metynnis sp., Mylesinus sp.) e sardinha (Triportheus elongatus). As espécies de plantas estudadas apresentam potencial de aproveitamento como fonte de alimento para peixes.

Palavras-chave: várzea, produção de frutos, peixe frugívoro

Fruit Production from the Floodplain Forest of Central Amazonia: a Food Resource for Fish

ABSTRACT - In order to quantify the contribution of four Central Amazon floodplain trees, Cassia leiandra Benth., Crescentia amazonica Ducke, Macrolobium acaciifolium (Benth.) Benth. and Vitex cymosa Bert. ex Spreng., as food resource for fishes, ten adult individuals of each species were labeled on Marchantaria Island. The peak of fruit offer occurred during the high water season, between the months of January and May. C. leiandra showed the biggest fruit production with $1,836 \mathrm{~kg} / \mathrm{ha}$ and greatest economic interest as a food resource for fishes, birds, monkeys and local people. The principal fruit consuming fishes were, tambaqui (Colossoma macropomum), matrinxã (Brycon cephalus), pirapitinga (Piaractus brachypomus), pirarara, (Phractocephalus hemioliopterus), bacu (Lithodoras dorsalis), pacu (Mylossoma sp., Myleus sp., Metynnis sp., Mylesinus sp.) and sardinha (Triportheus elongatus). The tree species show potential as a food resource for fishes.

Key-words: varzea, fruit production, frugivorous fish

\section{INTRODUÇÃ O}

As várzeas são formadas por florestas inundáveis e apresentam uma grande diversidade de espécies arbóreas e peixes. Os rios que inundam estas áreas tem a hidroquímica das águas classificadas como "branca", ricas em minerais dissolvidos, grande quantidade de partículas em suspensão e $\mathrm{pH}$ neutro (Sioli,1984; Furch, 1997). A inundação é regular, uma vez ao ano, monomodal e tem uma amplitude de 5 a $15 \mathrm{~m}$ acima do nível do mar, que pode mudar ao longo do curso do rio (Junk, 1985; 1998). A alta concentração de nutrientes dissolvidos e a grande carga sedimentar fértil, conferem a este ecossistema,

${ }^{1}$ (CPBO-INPA), ${ }^{2}$ (PIBIC-INPA/CPBO). Alameda Cosme Ferreira, 1756. Caixa Postal, 478. CEP 60011-970. Mauaus, Amazonas - Bresil. e-mail: alencar@inpa.gor.br 
fertilidade e alta produtividade. Estas florestas são importantes à dieta alimentar de muitas espécies de peixes, que durante a cheia penetram nestes ambientes, onde se alimentam de folhas jovens, frutos, sementes etc., que caem na água (Maia,1997). O alimento básico de muitas destas espécies são os frutos e as sementes (Honda, 1974; Saint-Paul, 1982; Zimburski, 1990; Silva, 1996; Maia 1997, 2001; Waldhoff \& Maia, 2000).

A bioprodutividade da várzea, em especial a produção de frutos, é item importante na cadeia alimentar e manutenção dos estoques pesqueiros. Foram encontradas mais de 500 árvores $\mathrm{ha}^{-1} \mathrm{e}$ a maioria destas produzem frutos e sementes que são consumidos por peixes (Campbell et al., 1992; Balslev et al., 1987; Foster,1990). Os frutos de Eschweilera tenuifolia e Hevea spruceana são ingeridos principalmente por Brycon cepahlus e Brycon sp. "matrinxã" e Colossoma macropomum "tambaqui" (Maia, 1997). Macrolobium multijugum tem especial importância na alimentação de $C$. macropomum e Podocnemis expansa "tracajá “ (Maia et al., 1998). Astrocaryum jauari, palmeira que ocorre às margens dos rios de águas preta e branca (igapó e várzea) contribui com uma biomassa significativa à dieta dos peixes, colocando a disposição 1,65 toneladas por hectare por ano de frutos frescos (Piedade, 1985). Myrciaria dubia, no igapó, apresenta dados em torno de 9,5 a 12,7 toneladas por hectare por ano de frutos frescos (Peters e Vasquez, 1987).

A produção anual de frutos nas florestas inundáveis da Amazônia varia entre 16 e 53 milhões de toneladas por ano (Waldhoff et al., 1996). Baseado na oferta de frutos destas florestas, estima-se a produção de 132.000 a 610.000 toneladas de peixe por ano na várzea (Goulding $e t$ al., 1996).

A produção média anual de frutos, neste ambiente apresenta considerável interesse quanto ao conhecimento da quantificação, freqüência de oferta e contribuição alimentar que possam proporcionar à fauna terrestre e aquática. Assim, a finalidade deste estudo foi de apresentar dados relativos à quantidade e contribuição que as espécies Cassia leiandra Benth., Crescentia amazonica Ducke, Macrolobium acaciifolium (Benth.) Benth. e Vitex cymosa Bert. ex Spreng., oferecem através de seus frutos à alimentação dos peixes, bem como identificar o potencial de aproveitamento e os principais peixes consumidores.

\section{MATERIAL E MÉTODOS}

A área de estudo localiza-se no lago Camaleão, Ilha da Marchantaria (MA) rio Solimões, $3^{\circ} 15^{\prime}$ Sul e $59^{\circ} 58^{\prime}$ Oeste (Fig. 1) a aproximadamente 20 $\mathrm{km}$ da cidade de Manaus, Amazonas. A área foi amostrada em 1,5 hectares, dividida em parcelas de 30X100 metros, margeando $500 \mathrm{~m}$ o lago e seguindo 30 $\mathrm{m}$ em direção a parte mais alta, entre as cotas 20 e 24 metros acima do nível do mar. Segundo Junk (1989), esta região pode permanecer em média entre 288 e 202 dias inundada. Foram contados 
todos os indivíduos das espécies estudadas com altura $\geq 2,0 \mathrm{~m}$ que frutificaram e mantiveram os frutos até o estágio maduro.

A vegetação em estudo está sujeita às flutuações do rio. A Figura 2 mostra a curva padrão, monomodal, da flutuação anual do rio Negro, cotas no porto de Manaus/AM, para 1988 e 1999.

Estimativa da produção de frutos

O estudo foi realizado no período de janeiro de 1998 a dezembro

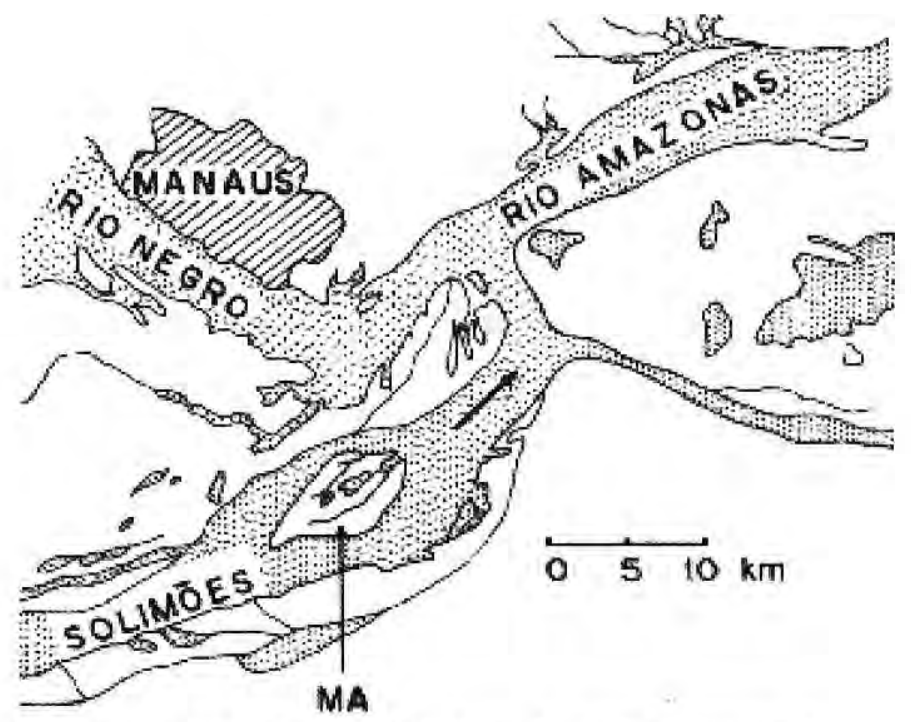

Figura 1. Área de estudo, (MA) Ilha da Marchantaria, $3^{\circ} 15^{\prime}$ S e $59^{\circ} 59^{\prime} \mathrm{O}$.

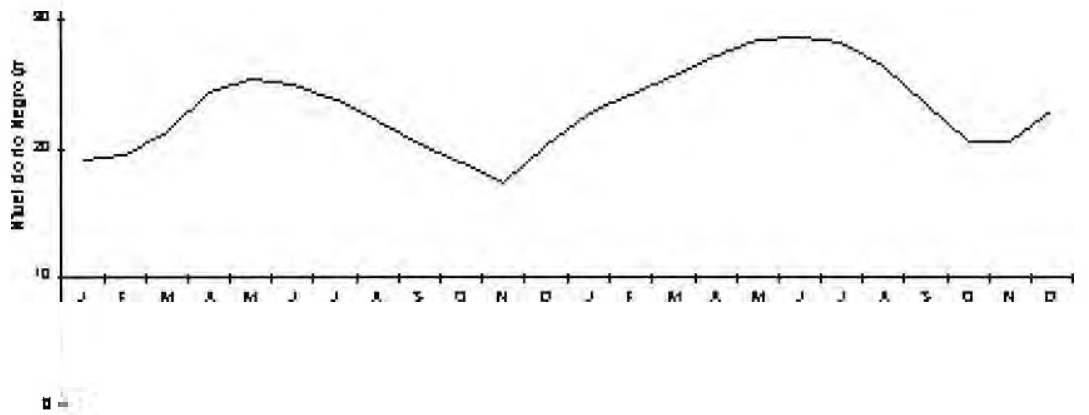

Figura 2. Flutuaçōes do nível do rio Negro em cotas sobre o nível do mar (a.n.m.) fornecidas pela PORTOBRAS, Capitania dos Portos Manaus, para os anos de 1998 e 1999. 
de 1999. A análise quantitativa de $C$. leiandra e C. amazonica foi estimada segundo Maia (1997) durante o pico de frutificação, a primeira entre janeiro e março e a segunda entre fevereiro e maio. As espécies em estudo frutificam uma vez ao ano. Foram marcados aleatoriamente 10 indivíduos de cada espécie dentro da área de estudo. Destes foram coletados todos os frutos maduros, com a mão ou com auxílio de varas (não foram considerados os frutos abortados). Os frutos foram coletados em duas etapas: na primeira foram retirados todos os frutos maduros e na segunda 30 dias após a primeira etapa, foram retirados os frutos restantes. Usou-se este procedimento para que a amostragem não fosse influenciada pela presença de frutos ainda imaturos e para que não houvessem perdas de frutos maduros, que provavelmente cairiam na água se não fossem coletados em duas etapas. Para a escolha da fase fruto maduro, foi estabelecido o período imediatamente anterior a abscissão (Maia, 1997). Após a coleta os frutos foram contados, pesados para obtenção de peso fresco e levados à secagem para obtenção do peso seco. A secagem foi feita em estufa a $60^{\circ} \mathrm{C}$, até ser obtido peso constante, neste caso ao redor de 7 dias. Para as espécies $M$. acaciifolium e $V$. cymosa, por apresentarem frutos pequenos, a determinação da produção por indivíduo foi feita através da coleta de todos os frutos em 3 galhos sorteados aleatoriamente. O valor médio da produção de fruto por galho foi multiplicado pelo número total de galhos de cada indivíduo. A coleta de $M$. acaciifolium se deu entre janeiro e abril e $V$. cymosa entre maio e julho. Em seguida os frutos, foram contados, pesados em balança (eletrônica Filizola CS-15) e secos em estufa a $60^{\circ}$ C até a estabilização do peso para posterior determinação do peso seco.

As informações relacionadas aos consumidores de frutos foram obtidas através de levantamento no herbário do INPA, consulta aos pescadores, conteúdo estomacal (3 tambaquis, 5 matrinchãs, 3 pirararas, 2 pirapitingas, 10 pacus, 10 bacu e 10 sardinhas) e citações literárias (Gottsberger, 1978; Smith, 1979; Goulding, 1980; SaintPaul, 1982; Zimburski, 1990; Maia, 1997, 2001; Lima \& Goulding,1998; Waldhoff \& Maia, 2000).

\section{RESULTADOS}

Na tabela 1 encontram-se o número de indivíduos/ha, a análise quantitativa da produção de frutos por espécie, em peso fresco e seco $(\mathrm{kg} / \mathrm{ha})$ e o teor de água (\%).

As cinco espécies estudadas totalizaram 259 indivíduos/ha. $V$. cymosa apresentou o maior número de indivíduos/ha (103), seguida por $C$. leiandra com 85/ha. As espécies $M$. acaciifolium e $C$. amazonica apresentaram menor quantidade, sendo 46 e 10 indivíduos/ha, respectivamente.

A produção total de frutos das espécies foi de $3.148 \mathrm{~kg} / \mathrm{ha}$ de peso fresco e $1.190 \mathrm{~kg} / \mathrm{ha}$ de peso seco. A maior produção de frutos em peso fresco, foi de $C$. leiandra com cerca de $1.836 \mathrm{~kg} / \mathrm{ha}$ e média de $22 \mathrm{~kg} /$ indivíduo, seguida por $M$. 
Tabela 1. Espécie vegetal, número de indivíduos/ha; analise quantitativa da produção de frutos $(n=10)$, produção média de fruto por indivíduo e desvio padrão, peso fresco e seco $(\mathrm{kg} / \mathrm{ha})$ e o teor de água (\%).

\begin{tabular}{lccccc}
\hline Espécie & Individuos/ha & $\begin{array}{c}\text { Produção media por indivi- } \\
\text { duo e desvio padrão }\end{array}$ & $\begin{array}{c}\text { Peso fresco } \\
(\mathrm{kg} / \mathrm{ha})\end{array}$ & $\begin{array}{c}\text { Peso } \\
\text { seco(kg/ha) }\end{array}$ & $\begin{array}{c}\text { teor de } \\
\text { água(\%) }\end{array}$ \\
\hline C. leiandra & 85 & $21,6 \pm 10,6$ & 1.836 & 546,6 & 70,7 \\
C. amazonica & 10 & $1,5 \pm 1.5$ & 15 & 5,3 & 64,6 \\
M. acaciifolium & 46 & $20,7 \pm 7,9$ & 950 & 541,5 & 43,0 \\
V. cymosa & 103 & $2,1 \pm 1,2$ & 227 & 27,3 & 88,0 \\
\hline
\end{tabular}

acaciifolium com $950 \mathrm{~kg} / \mathrm{ha}$ e média $21 \mathrm{~kg}$ /indivíduo, V. cymosa $227 \mathrm{~kg} /$ ha e $2,5 \mathrm{~kg} /$ indivíduo e C. amazonica $15 \mathrm{~kg} / \mathrm{ha}$ e $1,5 \mathrm{~kg} /$ indivíduo.

Os frutos apresentaram um teor de água médio de $61 \%$. O maior teor de água foi encontrado em $V$. cymosa com $88 \%$, seguida por $C$. leiandra com 70,7\%, C. amazonica $64,6 \%$ e $M$. acaciifolium $43 \%$.

$O$ pico de produção de frutos para as espécies estudadas, período de maior oferta alimentar, ocorreu entre os meses janeiro e maio de cada ano (1998 e 1999), durante a fase aquática.

A espécie $C$. leiandra apresentou excelente potencial econômico, uma vez que é amplamente consumida pela população local, pássaros (arara e papagaio), macacos e peixes. Segundo observações de conteúdo estomacal e pescadores, os principais peixes consumidores foram: bacu (Lithodoras dorsalis, Megalodoras sp.), matrinxã (Brycon cephalus), pacu (Myleus sp., Metynnis), pirapitinga (P. brachypomus) e tambaqui (C. macropomum)

$M$. acaciifolium apresentou importante contribuição na cadeia alimentar de peixes e quelônios.
Informações de herbário, pescadores e conteúdo estomacal, indicaram como principais consumidores: quelônios, tracajá (Podocnemis unifilis) e tartaruga (Podocnemis expansa); os peixes, piranha (Pygocentrus sp., Serrasalmus sp.), pirarara (Phractocephalus hemioliopterus), pirapitinga ( $P$. brachypomus), tambaqui (C. macropomum) e os pássaros (arara e papagaio).

$V$. cymosa foi consumida por pássaros, macacos e peixes. Os peixes consumidores foram bacu ( $L$. dorsalis), matrinxã (B. cephalus), pacu (Mylossoma sp., Myleus sp., Metynnis sp., Mylesinus sp.), pirapitinga ( $P$. brachypomus), sardinha (Triportheus elongatus) e tambaqui (C. macropomum) segundo informações de herbário, conteúdo estomacal e pescadores.

C. amazonica foi utilizada por matrinxã (B. cephalus), pacu (Mylossoma sp., Myleus sp., Metynnis sp., Mylesinus sp., pirarara $(P$. hemioliopterus), pirapitinga ( $P$. brachypomus), sardinha (Triportheus elongatus) e tambaqui ( $C$. macropomum), segundo informações dos pescadores e conteúdo estomacal. 
Os principais peixes consumidores entre as espécies estudadas foram: tambaqui ( $C$. macropomum), matrinxã cephalus), pirapitinga brachypomus), pirarara $(B$ $(P$ hemioliopterus), pacu (Mylossoma sp., Myleus sp., Metynnis sp., Mylesinus sp.) e sardinha (Triportheus elongatus) tabela 2.

\section{DISCUSSÃO}

Apesar da falta de dados mais precisos sobre a área inundável da Bacia Amazônica, estima-se que cerca de $20 \%$ desta são áreas inundáveis (Junk,1993; 1998). Considerando que as várzeas do rio Amazonas e tributários representam cerca de $200.000 \mathrm{~km}^{2}$ e que a sua bioprodutividade, em especial a produção de frutos, é item importante na cadeia alimentar e manutenção dos estoques pesqueiros, as espécies $C$. leiandra, C. amazonica, $M$. acaciifolium e $V$. cymosa, apresentaram potencial de aproveitamento. Entre estes, destacaram-se a produção de alimentos para animais aquáticos e terrestres, salientando a importante contribuição na alimentação de peixes.

$O$ peixe representa parcela considerável na dieta alimentar do amazônida constituindo uma das principais fontes de proteína animal e suporte econômico da região através da comercialização do pescado. Portanto, as espécies estudadas contribuem indiretamente à alimentação da população local. $C$. leiandra (com a produção de frutos de $1.836 \mathrm{~kg} / \mathrm{ha}$, peso fresco) além de apresentar importante papel na

Tabela 2. Lista dos principais peixes consumidores e produtores de frutos, número de estômagos examinados por espécie e porcentagem de estômagos com frutos.

\begin{tabular}{|c|c|c|c|}
\hline $\begin{array}{l}\text { CONSUMIDOR } \\
\text { Nome vulgar }\end{array}$ & $\begin{array}{l}\text { PRODUTOR DE FRUTOS } \\
\text { Nome cientifico }\end{array}$ & $\begin{array}{l}\text { Conteúdos } \\
\text { estomacais } \\
\text { examinados }\end{array}$ & $\begin{array}{l}\text { Estômagos com } \\
\text { frutos }(\%)\end{array}$ \\
\hline bacu & C. leiandra, V. cymosa, & - & - \\
\hline matrinxã & C. leiandra, V. cymosa, C. amazonica & 5 & 100 \\
\hline pacu. & C. leiandra, V. cymosa, C. amazonica & 10 & 100 \\
\hline piranha & M. acacilfolium & - & - \\
\hline pirapitinga & C. leiandra, V. cymosa, C. amazonica, M. acacilfolium & 2 & 100 \\
\hline pirarara & M. acaciifolium, C. amazonica & 3 & 100 \\
\hline sardinha & V. cymosa, C.amazonica & 10 & 80 \\
\hline tambaqui & C. leiandra, V. cymosa, C. amazonica, M. acacilfolium & 3 & 100 \\
\hline tartaruga & M. acacifolium & _ & - \\
\hline tracajá & M. acacifolium & _ & _ \\
\hline
\end{tabular}

bacu (Lithodoras dorsalis, Megalodoras sp.); matrinxä (Brycon cephalus); pacu (Mylossoma sp., Myleus sp., Metynnis sp., Mylesinus sp.); piranha (Pygocentrus sp.); pirapitinga (Piaractus brachypomum); pirarara (P. hemioliopterus); sardinha (Triportheus elongatus); tambaqui (Colossoma macropomum); tartaruga (Podocnemis expansa), tracaja (Podocnemis unifilis). 
alimentação de peixes, tem excelente potencial econômico, podendo ser comercializada nas feiras, pois é amplamente consumida na região.

As várzeas do rio SolimõesAmazonas e dos seus afluentes apresentam potencial produtivo para a pesca, a agricultura e a silvicultura (Junk, 1998). Com o aumento demográfico a utilização destas áreas tem se intensificado e em algumas áreas é visível a degradação do ecossistema. Vários estudos tem abordado a ecologia e o aproveitamento deste ambiente, destacando-se os seguintes: Smith (1981), Junk (1985, 1993, 1998), Bayley \& Petrere (1989), Goulding et al (1996) e Maia (2001).

O número de espécies de peixes na Amazônia que se alimentam de frutos e sementes é grande (Gottsberger, 1978; Smith, 1979; Goulding, 1989; Ziburski, 1990; Maia, 1997; Lima \& Goulding, 1998; Waldhoff \& Maia, 2000; Maia, 2001). Entre estes, o tambaqui é uma espécie nobre, de alto valor comercial. Nos centros urbanos, grande parte da população não tem poder aquisitivo para adquiri-lo. Outros peixes como cará, matrinxã, pacu, pirapitinga, pirarara e sardinha, são espécies de valor comercial inferior e portanto mais consumidos pela maioria da população regional.

A variação na produção de frutos por indivíduo, das espécies estudadas, parece relacionada à idade do vegetal. Segundo Maia (1997) Hevea spruceana, espécie de igapó, apresentou uma maior produção de frutos entre os 15 e 45 anos; a partir de 45 anos iniciou o declínio na produção. Porém, as causas da variação na produção de frutos das espécies estudadas, só poderão ser conhecidas após um estudo da produção de frutos em relação à idade do vegetal.

$O$ teor de água (média total) nos frutos de $61 \%$ nas espécies estudas foi considerado baixo se comparado à maioria dos frutos utilizados no consumo humano. Entretanto, grande parte dos frutos ingeridos por peixes tem baixo teor de água e carboidrato solúvel, mas alto teor de proteínas e gordura (Waldhoff et al.,1996).

$O$ período de maior oferta de alimentos, no caso da produção de frutos, se deu na fase aquática, entre janeiro e maio. A produção total de frutos para as quatro espécies, de 3.148 $\mathrm{kg} / \mathrm{ha} / \mathrm{ano}$ de peso fresco, mostrou a importância destes vegetais, que além de contribuírem como fonte de alimento para os peixes, podem ser inseridos no item recuperação ambiental e utilizados na regeneração de áreas inundáveis degradadas, pois ao maximizarem a cobertura vegetal, restauram a oferta de alimentos (Maia, 2001).

A contribuição alimentar de algumas espécies na alimentação de peixes, não se restringe a produção de fruto; a oferta de alimento pode ser superior, uma vez que folhas jovens, flores e frutos abortados também contribuem à dieta (Maia, 1997). Por outro lado, algumas espécies que apresentam alta produção fruto têm o maior peso na casca, que às vezes não é comestível para os peixes. Por exemplo Pseudobombax munguba, apenas as sementes são comestíveis 
(Chalco, 2001). Neste estudo, verificou-se que os frutos de $M$. acaciifolium e $V$. cymosa foram consumidos integralmente; de $C$. amazonica e $C$. leiandra foram consumidas as sementes.

A produção anual de frutos que entra no sistema das áreas inundáveis da Amazônia é praticamente desconhecida. A avaliação da produção de frutos por hectare/ano, por alguns autores, para a área total da Amazônia é prematura e deve ser mais criteriosa. Tais estimativas só poderão ser elaboradas quando houver uma amostragem representativa de levantamento florístico, número de espécies e produção média de frutos por indivíduo por hectare por ano, em diferentes pontos da planície de inundação.

\section{AGRADECIMENTOS}

As autoras agradecem ao $\mathrm{CNPq}$, Prog.SHIFT/ENV-29, Proj. INPA/ Max-Planck e PP-G7/PPD-1161-99 pelo apoio financeiro e aos técnicos da CPBO, José Guedes de Oliveira, José Ramos e Felipe França, pela participação nos trabalhos de campo.

\section{Bibliografia citada}

Balslev, H.; Luteyn. J.; Ollgaard,B., 1987. Composition and struture of unflooded and floodplain forest in Amazonian Ecuador. Opera Botanica, 92: 37-57.

Bayley, P.B.; Petrere Jr., M. 1989. Amazon fisheries: assessment methods, current status, and management options. In: Dodge, D.P. (ed.): Proceedings of the International

Large River Symposium. Canada. Special Publication. Fish. Aquat. Sci, 106:385-398.

Campbell, D. G.; Stone, J.L.; Rosas, A. 1992. A comparision of the phytosociology and dynamics of three floodplain (várzea) for- ests of known age, Rio Juruá, western

Brazilian Amazon. Bot. J. Linn. Society, 108: 213-237.

Chalco, F.P. 2001. Produção de Frutos de Espécies Arbóreas Importantes na Alimentação de Peixes - Amazônia Central. Monografia apresentada para obtenção do grau Eng. Florestal. UTAM, Manaus, AM. $36 \mathrm{p}$.

Foster, R. 1990. The floristic composition of the Rio Manú floodplain forest. Four Neotropical Rainforests. New Haven.

Furch, K. 1997. Chemistry of várzea and igapó soils and nutrient inventory of their floodplains. In: Junk,W.J. (ed.). The Central Amazon floodplain. Ecology of a pulsing

system. Springer, Ecological Studies, 126:47-67.

Gottsberger, G. 1978. Seed dispersal by fish in the inundated regions of Humaitá, Amazonia. Biotropica, 10:170-183.

Goulding, M. 1980. The Fishes and Forest: Explorations in Amazonian Natural History. University of California Press, Berkeley, USA. 280 p.

Goulding, M. 1985. Forest fishes of the Amazon. In: Prance, GT.; Lovejoy, T.E. (Eds.). Amazonia. Pergamon Press, Oxford, p. 267-276.

Goulding, M.; Smith, N.J.H.; Mahar, D.J. 1996. Flood of Fortune. Ecology and Eeconomy Along the Amazon, New York.

Honda, E.M.S. 1974. Contribuição ao conhecimento da biologia de peixes do Amazonas II-Alimentação do tambaqui, Colossoma bidens. Acta Amazônica, 4: 47-53.

Junk,W.J.1985. The Amazon floodplain - A sink or source for organic carbon? Mitt.Geol. Paläont. Inst. Univ. Hamburg. SCOPE/UNEP Sonderband Heft 58. Hamburg. p. 267-283.

Junk, W.J. 1993. Wetlands of tropical South America. In: Whigham, D.F. et al (Eds.). Wetlands of the World I. Kluwer Academic Publishers. Netherlands. p. 679-739.

Junk, W.J. 1998. A várzea do Rio Solimões Amazonas: Conceito para o aproveitamento sustentável de seus 
recursos. In: Anais do IV Simpósio de Ecossistemas Brasileiros. Àguas de Lindóia-SP. p.1-24.

Lima, C. A. \& M. Goulding, 1998. Os Frutos do Tambaqui: Ecologia, Conservação e

Cultivo na Amazônia. Sociedade Civi] Mamirauá. Editora Lithera Maciel Ltda.

Maia, L.M.A, 1997. Influência do Pulso de Inundação na Fisiologia, Fenologia e Produçâo de Frutos de Hevea spruceana (Euphorbiaceae) e Eschweilera tenuifolia (Lecythidaceae), em Área Inundável de Igapó da Amazônia Central. Tese de Doutorado, INPA/FUA. Manaus, AM. $195 \mathrm{p}$.

Maia, L.M.A.; Piedade, M.T.F.; Soares, M.G. 1998. The phenology of Macrolobium multijugum (Caesalpiniaceae) in flooded forest, in the black water (igapo) of the Tarumã - Mirim, Central Amazonia, Brazil. In: Proceedings of the Third SHIFT Workshop. Manaus-AM. p. 293-303.

Maia, L.M.A. 2001. Frutos da Amazônia: Fonte de Alimento para Peixes. SEBRAE$A M$. Manaus, AM, 143 p.

Peters, C. M.; Vasquez, A. 1987. Estudios ecológicos de camu-camu (Myrciaria dubia) I. Producción de frutos en poblaciones naturales. Acta Amazônica, 16/17 (n.único):161-174.

Piedade, M.T.F. 1985. Ecologia e Biologia Reprodutiva de Astrocaryum janari Mart. (Palmae) como Exemplo de População Adaptada as Áreas Inundáveis do Rio Negro (igapós). Dissertação de Mestrudo, INPA/FUA. Manaus, AM. 187 p.

RADAMBRASIL. 1978. Levantamento de Recursos Naturais. Folha SA 20 Manaus. Rio de Janeiro, Departamento Nacional de Produção Mineral.

Revilla, J.D.C. 1981. Aspectos Florísticose Fitossociológicos da Floresta Inundável (igapó). Praia Grande, Rio Negro, Amazonas, Brasil. Dissertação de Mestrado, INPA/FUA. Manaus, AM. 129 p.
Saint-Paul, U. 1982. Okologische und Physiologische Untersuchngen an dem Amazonnasfisch Colossoma macropomum (Cuvier 1818) im Hinblick auf seine Eignung fiir die Tropische Fischzucht (Pisces Serrasalmidae). Ph.D. Thesis. Fachbereiches Biologie der Universität Hamburg. Hamburg. 220 p.

Silva, J.A. Moreira da. 1996. Nutrientes, Energia e Digestibilidade Aparente de Frutos e Sementes Consumidos pelo tambagui (Colossoma macropomum Cuvier) nas florestas inundáveis da Amazônia Central. Tese de doutorado. INPA/FUA. Manaus, AM. 114 p.

Sioli, H. 1984. The Amazon and its main affluentes: Hydrography Morphology of the River Courses and River Types. In: Sioli, H. (Ed.). The Amazon: Limnology and Landscape Ecology of a Mighty Tropical River and its Basin. Dr. W. Junk Publisher, Dordrecht. Netherlands. p.127-165.

Smith, N.J.H. 1979. A pesed no Rio Amazonas. CNPq/INPA. Manaus-AM. 154 p.

Smith, N.J.H. 1981. Man, fishes and the Amazon - Columbia University Press, New York.

Zimbusrski, A. 1990. Ausbreitungs und Reproduktionsbiologie Einiger Baumarten Der Amazonischen Überschwemmungswälder. Ph.D. Thesis. Fachbereiches Biologie der Universität Hamburg. Hamburg. 112 p.

Waldhoff, D.; U.Sait-Paul; B.Furch. 1996. Value of fruits and seeds from the floodplain forests of Central Amazonia as food resource for fish. Ecotropica 2:143- 156.

Waldhoff, D. \& Maia, L.M.A. 2000. Production and chemical composition of fruits from trees in flox)dplain forest of Central Amazonia and their importance for fish. In: Junk, W.J., Ohly, J., Piedade, M.T.F. \& Soares, M.G. (eds.): The Central Amazon floodplain: Actual Use and Options for a Sustainable Management. Backhuys Publishers, Leiden, The Netherlands, pp. 393-415. 
recursos. In: Anais do IV Simpósio de Ecossistemas Brasileiros. Àguas de Lindóia-SP. p. 1-24.

Lima, C. A. \& M. Goulding, 1998. Os Frutos do Tambaqui: Ecologia, Conservação e

Cultivo na Amazônia. Sociedade Civil Mamirauá. Editora Lithera Maciel Ltda.

Maia, L.M.A, 1997. Influência do Pulso de Inundação na Fisiologia, Fenologia e Produção de Frutos de Hevea spruceana (Euphorbiaceae) e Eschweilera tenuifolia (Lecythidaceae), em Área Inundável de Igapó da Amazônia Central. Tese de Doutorado, INPA/FUA. Manaus, AM. 195 p.

Maia, L.M.A.; Piedade, M.T.F.; Soares, M.G. 1998. The phenology of Macrolobium multijugum (Caesalpiniaceae) in flooded forest, in the black water (igapo) of the Tarumã - Mirim, Central Amazonia, Brazil. In: Proceedings of the Third SHIFT Workshop. Manaus-AM. p. 293-303.

Maia, L.M.A. 2001. Frutos da Amazônia: Fonte de Alimento para Peixes. SEBRAE$A M$. Manaus, AM, 143 p.

Peters, C. M.; Vasquez, A. 1987. Estudios ecológicos de camu-camu (Myrciaria dubia) I. Producción de frutos en poblaciones naturales. Acta Amazônica, 16/17 (n.único):161-174.

Piedade, M.T.F. 1985. Ecologia e Biologia Reprodutiva de Astrocaryum jauari Mart. (Palmae) como Exemplo de População Adaptada as Áreas Inundáveis do Rio Negro (igapós). Dissertação de Mestrado. INPA/FUA. Manaus, AM. 187 p.

RADAMBRASIL. 1978. Levantamento de Recursos Naturais. Folha SA 20 Manaus. Rio de Janeiro, Departamento Nacional de Produção Mineral.

Revilla, J.D.C. 1981. Aspectos Florísticos e Fitossociológicos da Floresta Inundâvel (igapó). Praia Grande, Rio Negro, Amazonas, Brasil. Dissertação de Mestrado, INPA/FUA. Manaus, AM. 129 p.
Saint-Paul, U. 1982. Ökologische und Physiologische Untersuchngen an dem Amazonnasfisch Colossoma macropomum (Cuvier 1818) im Hinblick auf seine Eignung fiur die Tropische Fischzucht (Pisces Serrasalmidae). Ph.D. Thesis. Fachbereiches Biologie der Universität Hamburg. Hamburg. 220 p.

Silva, J.A. Moreira da. 1996. Nutrientes, Energia e Digestibilidade Aparente de Frutos e Sementes Consumidos pelo tambaqui (Colossoma macropomum Cuvier) nas florestas inundáveis da Amazônia Central. Tese de doutorado. INPA/FUA. Manaus, AM. 114 p.

Sioli, H. 1984. The Amazon and its main affluentes: Hydrography Morphology of the River Courses and River Types. In: Sioli, H. (Ed.). The Amazon: Limnology and Landscape Ecology of a Mighty Tropical River and its Basin. Dr. W. Junk Publisher, Dordrecht, Netherlands. p.127-165.

Smith, N.J.H. 1979. A pesca no Rio Amazonas. CNPq/INPA. Manaus-AM. 154 p.

Smith, N.J.H. 1981. Man, fishes and the Amazon - Columbia University Press, New York.

Zimbusrski, A. 1990. Ausbreitungs und Reproduktionsbiologie Einiger Baumarten Der Amazonischen Überschwemmungswälder. Ph.D. Thesis. Fachbereiches Biologie der Universität Hamburg. Hamburg. 112 p.

Waldhoff, D.; U.Sait-Paul; B.Furch. 1996. Value of fruits and seeds from the floodplain forests of Central Amazonia as food resource for fish. Ecotropica 2:143- 156.

Waldhoff, D. \& Maia, L.M.A. 2000. Production and chemical composition of fruits from trees in floodplain forest of Central Amazônia and their importance for fish. I $n$ : Junk, W.J., Ohly, J., Piedade, M.T.F. \& Soares, M.G. (eds.): The Central Amazon floodplain: Actual Use and Options for a Sustainable Management. Backhuys Publishers, Leiden, The Netherlands, pp. 393-415. 